\title{
Validação da versão brasileira do questionário genérico de qualidade de vida short-form 6 dimensions (SF-6D Brasil)
}

\author{
Validation of the brazilian version of the generic six-dimensional \\ short form quality of life questionnaire (SF-6D Brazil)
}

\author{
Alessandro Gonçalves Campolina ${ }^{1}$ \\ Adriana Bruscato Bortoluzzo ${ }^{2}$ \\ Marcos Bosi Ferraz ${ }^{1}$ \\ Rozana Mesquita Ciconelli ${ }^{1}$
}

\footnotetext{
${ }^{1}$ Universidade Federal de São Paulo. Rua Botucatu 740, Vila Clementino. 040023-062 São Paulo SP. alecampolina@gmail.com ${ }^{2}$ Insper - Instituto de Ensino e Pesquisa.
}

\begin{abstract}
Objective: This study seeks to present the translation and validation of the SF- $6 D$ in the Brazilian context, based on the model and the version developed in the United Kingdom in 2002. Methods: An observational and transversal study. The tools for assessing quality of life were applied, namely HAQ, SF-36, EQ-5D and SF-6D (2002 version). Descriptive statistics and correlation coefficients were used for data analysis. Results: 200 patients with rheumatoid arthritis, with a mean age of 49.22 years, 11.16 years of disease and mean HAQ 1.02 were studied. Preferences measured by the SF-6D, the EQ-5D and the techniques of EVA, TTO and SG were found to correlate among themselves, with Pearson coefficients from 0.19 to $0.66(p<0.01)$. Conclusion: The SF$6 D$ Brazil questionnaire represents a valid option for assessing preferences on economic analysis conducted in Brazil.
\end{abstract}

Key words Rheumatoid arthritis, Quality of life, Health economics
Resumo Objetivo: O presente estudo tem por objetivo apresentar a tradução e a validação do questionário SF-6D (Short-Form 6 dimensions) para o contexto brasileiro, com base no modelo e na versão desenvolvida no Reino Unido em 2002. Métodos: Estudo observacional e transversal. Foram aplicados os instrumentos de avaliação de qualidade de vida: HAQ (Health Assessment Questionaire), SF-36, EQ-5D (EuroQol-5D) e SF-6D (versão de 2002). Estatísticas descritivas e coeficientes de correlação foram usados para a análise dos dados. Resultados: Foram avaliados 200 pacientes portadores de artrite reumatóide, com média de idade de 49,22 anos, tempo medido de doença de 11,16 anos e HAQ médio de 1,02. As preferências mensuradas pelo $S F-6 D$, pelo EQ-5D e pelas técnicas de EVA (escala visual analógica), TTO (time trade-off) e SG (standard gamble) apresentaram correlações significativas entre si, com coeficientes de Pearson variando de 0,19 a 0,66 ( $p<0,01)$. Conclusão: O questionário SF$6 D$ Brasil representa uma opção de questionário válido para a avaliação de preferências em análises econômicas realizadas em nosso meio.

Palavras-chave Artrite reumatóide, Qualidade de vida, Economia da saúde 


\section{Introdução}

Nos últimos anos, vários autores têm investigado a utilização do instrumento genérico de qualidade de vida "Medical Outcomes Study 36-Item Short-Form Health Survey” (SF-36) ${ }^{1}$ em análises econômicas de intervenções em saúde, por ser este um questionário avaliado em mais de 200 doenças e traduzido em 40 países ${ }^{2}$.

Para tanto, torna-se necessário que o nível de qualidade de vida dos sujeitos em estudo seja quantificado aplicando o conceito de preferência, com base na teoria da decisão sob incerteza publicada em 1944 por John von Neumann e Oscar Morgenstern, pela qual se entende que indivíduos têm preferências por diferentes estados de saúde ${ }^{3-4}$.

As preferências por estados de saúde podem ser mensuradas utilizando-se medidas diretas, que empregam técnicas de valoração e escolha, ou utilizando-se medidas indiretas que empregam questionários desenvolvidos a partir das técnicas anteriores.

Basicamente existem três técnicas mais utilizadas para a medida direta de preferências: o standard gamble (SG) ou escolha pela chance, o time trade-off (TTO) ou escolha pelo tempo e a escala visual analógica (EVA). Estas medidas são, entretanto, complexas e demandam processos de aplicação demorados e custosos ${ }^{3}$. Uma abordagem mais prática e que tem sido mais empregada, é a utilização de questionários que permitem descrever e calcular preferências para diversos estados de saúde. Entre os instrumentos mais conhecidos estão: o EuroQol-5D (EQ-5D), o Quality of WellBeing (QWB), o Health Utilities Index (HUI $)^{5-8}$.

A utilização do SF-36 para a mensuração de preferências em saúde já foi detalhada em seis publicações, que descrevem oito algoritmos diferentes para a derivação de preferências por estados de saúde a partir deste questionário ${ }^{9-12}$.

Como resultado destes esforços investigativos foi desenvolvido no Reino Unido o questionário genérico de qualidade de vida Short-Form 6 dimensions (SF-6D), que permite a obtenção de medidas de preferência por estados de saúde, a partir dos itens do SF-36,12.

No Brasil, este questionário já se encontra traduzido e validado em sua primeira versão, desenvolvida em $1998^{13}$. Entretanto, o novo modelo de construção do SF-6D, desenvolvido em 2002, ainda não se encontra disponível em nosso meio.

O presente estudo tem por objetivo apresentar a tradução e a validação do questionário SF-6D para o contexto brasileiro, com base no modelo e na versão desenvolvida no Reino Unido em 2002.

\section{Métodos}

\section{Delineamento de estudo e amostragem}

O delineamento de estudo adotado foi o observacional, transversal. A amostra foi selecionada no ambulatório de Reumatologia da Universidade Federal de São Paulo, a partir de um recorte temporal aleatório, de abril de 2005 a abril de 2006, para garantir um processo de amostragem probabilística por demanda. Os participantes foram selecionados em diferentes dias da semana para fins de representatividade temporal.

Foram incluídos pacientes com diagnóstico de artrite reumatóide, conforme os critérios do Colégio Americano de Reumatologia - $\mathrm{ACR}^{14}$, com idade maior que 18 anos e menor ou igual a 65 anos, em acompanhamento no serviço.

Foram excluídos pacientes que apresentam outras doenças reumáticas associadas, doenças psiquiátricas ou fibromialgia diagnosticadas e/ ou na vigência de tratamento para estas condições. Pacientes com déficit cognitivo grave que impossibilitem a compreensão dos instrumentos de pesquisa, também foram excluídos.

\section{Roteiro de aplicação \\ do protocolo de pesquisa}

Os instrumentos de coleta de dados do protocolo de pesquisa foram aplicados na seguinte ordem:

1) Técnicas de medida direta de preferências (EVA, TTO, SG), por escolha aleatória segundo tabela de randomização

2) SF-6D

3) $\mathrm{EQ}-5 \mathrm{D}$

4) HAQ

5) Questionário clínico e demográfico

6) Exame clínico

\section{Instrumentos de avaliação}

\section{O Questionário Short-Form 6 Dimension (SF-6D Brasil)}

Os itens do SF-36 usados para a construção do SF-6D (versão de 2002) foram capacidade funcional: itens 1,2 e 10; limitação global: item 3 de aspectos físicos e item 2 de aspectos emocionais; aspectos sociais: item 2; dor: todos os itens; saúde mental: item 1 e vitalidade: item 2 .

$\mathrm{Na}$ versão brasileira do questionário SF-6D (SF-6D Brasil), todos os itens foram extraídos 


\section{Health Assessment Questionaire (HAQ)}

O Health Assessment Questionaire (HAQ) é um instrumento específico de qualidade de vida desenvolvido para permitir a avaliação de parâmetros de estado de saúde em ensaios terapêuticos envolvendo pacientes com artrite reumatóide. A descrição do instrumento pode ser obtida em outra publicação ${ }^{16}$.

No presente estudo, este questionário foi aplicado através de entrevista padronizada, realizada por um pesquisador treinado.

\section{Euroqol-5D (EQ-5D)}

O Euroqol-5D (EQ-5D) é um instrumento desenvolvido na Europa para a mensuração indireta de preferências por estados de saúde. A descrição da ferramenta, bem como os procedimentos para a obtenção dos escores, encontrase detalhada em outras publicações ${ }^{17,18}$.

No presente estudo, este questionário foi aplicado através de entrevista padronizada, realizada por um pesquisador treinado.

\section{Escala Visual Analógica (EVA)}

Para a avaliação direta de preferências por estados de saúde, a técnica de EVA foi usada inicialmente para a criação de 3 cenários hipotéticos de saúde. Cada um dos cenários descreve um estado diferente de saúde, envolvendo os níveis de qualidade de vida: pobre, moderado e bom (com base nas questões do SF-36).

Os entrevistados foram solicitados a indicar quantas vezes um dado estado de saúde (cenário) foi pior em relação a um outro, em uma escala de 0 a 1 . Nesta escala, o valor 1 corresponde ao melhor estado de saúde possível e o valor 0 ao pior possível.

Depois de ancorar os cenários hipotéticos sobre a escala, os entrevistados foram convidados a pensar sobre o seu próprio estado de saúde naquele momento, e marcar um ponto sobre esta mesma escala que corresponderia ao valor de preferência para o seu próprio estado de saúde ${ }^{19}$.

\section{Time-Trade Off (TTO)}

$\mathrm{Na}$ técnica de TTO os respondentes foram solicitados a escolher ou ser indiferente entre duas alternativas para o seu estado de saúde, diante de uma intervenção em saúde hipotética.

Em uma alternativa, foi oferecido ao respondente a manutenção do seu estado de saúde para o resto da vida. Na outra alternativa, foi oferecido a ele um estado de saúde melhor por um período de tempo menor (por exemplo, boa qualidade de vida por 10 anos, seguido de morte imediata). Baseado nas respostas, o período de tempo menor foi variado até que se determinasse o ponto de indiferença, que seria o menor tempo com a máxima qualidade de vida possível, que faria o indivíduo abandonar sua condição intermediária de saúde e se submeter ao possível procedimento terapêutico.

Este ponto ou tempo de indiferença é o TTO para o estado de saúde atual do respondente, numa escala onde 0 significa morte imediata e 1 significa o estado de saúde melhor para o resto da vida.

Para este estudo, baseado em dados do DATASUS de 2003, assumimos uma expectativa de vida ao nascer de 73 anos para o sexo feminino e de 65 anos para o sexo masculino. Do mesmo modo, assumimos uma expectativa de vida aos 60 anos para o sexo feminino de 20 anos e para o sexo masculino de 16 anos (DATA-SUS, 2003).

Após a explicação da técnica, foram calculados os anos de vida remanescentes baseado na idade e expectativa de vida para a idade e sexo. Em seguida, a aplicação da técnica foi realizada em formato "ping-pong"19.

\section{Standard Gamble (SG)}

Na técnica de SG os respondentes foram solicitados a escolher entre duas alternativas oferecidas, diante de uma intervenção em saúde hipotética.

Em uma alternativa foi oferecido ao respondente um estado de saúde intermediário, ou seja, o seu estado de saúde sob condição de certeza. Em outra alternativa, foram oferecidos ao respondente dois estados de saúde sob condição de incerteza, um que seria o melhor possível, como, por exemplo, saúde perfeita (com probabilidade $\mathrm{p}$ de ocorrer, caso o paciente se submeta ao procedimento terapêutico) e um que seria o pior cenário possível, como, por exemplo, a própria morte (com probabilidade 1-p). Baseado nas respostas dadas, a probabilidade $\mathrm{p}$ foi variada até que se determinasse o ponto de indiferença 
$\mathrm{p}^{\star}$ (que seria um ponto em que o indivíduo seria indiferente entre se submeter a um procedimento capaz de trazer resultados incertos e permanecer em uma situação de certeza, no caso, o seu estado de saúde atual).

Este ponto de indiferença $\mathrm{p}^{*}$ é o SG do respondente para o seu estado de saúde intermediário apresentado inicialmente, numa escala em que o pior estado é 0 e o melhor é 1 .

O formato "ping-pong" de aplicação da técnica foi utilizado com o auxílio do dispositivo visual $^{19}$, que apresentava as seguintes probabilidades p de desfecho: 100\%, 99\%, 98\%, 97\%, 96\%, $95 \%, 90 \%, 85 \%, 80 \%, 70 \%, 60 \%, 50 \%$, е $0 \%$.

\section{Análise estatística e aspectos éticos}

O foco da análise foi a comparação das medidas obtidas com o SF-6D, com aquelas obtidas com EQ-5D, EVA, TTO e SG. A validade de construto do SF-6D é testada comparando os escores obtidos com a medida indireta de preferência empregada neste estudo (o EQ-5D) e as medidas diretas de preferência (EVA, TTO e SG), instrumentos estes, validados e amplamente utilizados em análises econômicas em saúde.

As análises foram realizadas através o pacote estatístico SPSS ${ }^{\circledast}$ Versão 11.0 para Windows ${ }^{\circledast}$. Estatísticas descritivas foram empregadas para a caracterização da amostra. As correlações entre as preferências medidas pelos modelos do SF6D, EQ-5D e as obtidas com as técnicas de EVA, TTO e SG foram determinadas utilizando o coeficiente de correlação de Pearson.

As correlações foram consideradas pequenas se variaram entre $0 \pm 2,5$; moderadas se variaram entre $\pm 0,26$ e $\pm 0,50$; boas se variaram entre \pm 0,51 e $\pm 0,75$; muito boas se $>0,75$ ou $<$ que $-0,75$.

Para este estudo, adotamos $\mathrm{p}<0,05$ (alfa $=$ $5 \%)$ para valores estatisticamente significativos.

Todos os participantes preencheram o termo de consentimento livre e esclarecido aprovado pelo Comitê de Ética em Pesquisa da Universidade Federal de São Paulo.

\section{Resultados}

Foram avaliados 200 pacientes que preenchiam os critérios da ACR para artrite reumatóide e que concordaram em participar do estudo. Nem todos os indivíduos selecionados conseguiram completar as avaliações por dificuldades de compreensão dos instrumentos de pesquisa.

Dos 200 indivíduos avaliados, 200 completaram o SF-6D e o EQ-5D, 199 completaram o HAQ e a EVA, 197 completaram o TTO e 188 completaram o SG.

A idade média dos participantes foi de 49,22 anos ( $\mathrm{DP}=10,0)$, sendo 78,0\% deles, pertencentes ao sexo feminino. A maior parte dos indivíduos referia apresentar cor de pele branca $(41,0 \%)$ e parda (56,5\%). A maioria era casada $(56,5 \%)$ e inativa no mercado de trabalho $(62,0 \%)$. A média dos anos de estudo foi de 6,38 anos (DP = $4,1)$; a média da renda per capita familiar foi de 366,88 reais $(\mathrm{DP}=367,6)$ e a média do número de habitantes por domicílio foi de 3,80 habitantes $(\mathrm{DP}=1,8)$, conforme a Tabela 1 .

O tempo médio de doença foi de 11,16 anos $(\mathrm{DP}=8,4)$, sendo que a maior parte dos participantes pertenciam às classes funcionais I e II (33,0\% e 38,5\%, respectivamente) e apresentavam um HAQ médio de 1,02. Setenta e quatro e meio por cento dos indivíduos não apresentavam manifestações extra-articulares e 73,0\% apresentavam deformidades articulares. No momento da avaliação, a média do número de articulações dolorosas foi de 5,56 e o de articulações edemaciadas foi de 7,35. A auto-avaliação de dor e de estado geral pela EVA teve uma média de 41,42 $\mathrm{mm}(\mathrm{DP}=25,1)$ e $67,30 \mathrm{~mm}(\mathrm{DP}=20,7)$, respectivamente, para os participantes do estudo ( Tabela 2).

A Tabela 3 apresenta a média dos domínios e sumários obtidos a partir do SF-36, as medidas de preferência obtidas com as técnicas de EVA, TTO e SG, e as medidas de preferência obtidas com os questionários EQ-5D e SF-6D.

A Tabela 4 revela correlações significativas ( $p$ $<0,01)$ entre as preferências obtidas com o SF6D, o EQ-5D e as técnicas de EVA, TTO e SG, com coeficientes de correlação variando de 0,19 a 0,66.

\section{Discussão}

Com o número crescente de análises econômicas publicadas na literatura científica, sem dúvida, o aprimoramento das ferramentas de medida tem sido objeto de especial interesse, inclusive no que se refere à praticidade de aplicação.

Os métodos classicamente empregados para a mensuração de preferências têm empregado técnicas trabalhosas e de difícil compreensão por parte dos pacientes, como a EVA, o TTO e o SG ${ }^{20}$. Estas técnicas têm tido sua aplicação questionada em populações com baixa escolaridade ou comprometimento cognitivo, em função dos vieses que podem produzir. Lenert e Kaplan chamam a atenção para o fato de que a habilidade cognitiva, a aversão ao risco e as habilidades nu- 
Tabela 1. Características sócio-demográficas dos pacientes com artrite reumatóide.

\begin{tabular}{|c|c|}
\hline n \% o & ou média (DP) \\
\hline \multicolumn{2}{|l|}{ Idade (anos) } \\
\hline $\mathrm{N}$ & 200 \\
\hline Média (DP) & $49,22(10,0)$ \\
\hline Mínimo - máximo & $20,00-65,00$ \\
\hline \multicolumn{2}{|l|}{ Sexo $(\mathrm{n} \%)$} \\
\hline Feminino & $156 / 78,0$ \\
\hline Masculino & $44 / 22,0$ \\
\hline \multicolumn{2}{|l|}{ Anos de estudo (anos) } \\
\hline $\mathrm{N}$ & 200 \\
\hline Média (DP) & $6,38(4,1)$ \\
\hline Mínimo - máximo & $0-15,00$ \\
\hline \multicolumn{2}{|l|}{ Renda per capita familiar (R\$) } \\
\hline $\mathrm{N}$ & 200 \\
\hline Média (DP) & $5,88(367,60)$ \\
\hline Mínimo - máximo $\quad 33,0$ & $00-3000,00$ \\
\hline \multicolumn{2}{|c|}{ Número de pessoas no domicílio } \\
\hline $\mathrm{N}$ & 200 \\
\hline Média (DP) & $3,80(1,8)$ \\
\hline Mínimo - máximo & $1,00-11,00$ \\
\hline \multicolumn{2}{|l|}{ Cor referida (n \%) } \\
\hline Branca & $82 / 41,0$ \\
\hline Parda & $79 / 39,5$ \\
\hline Preta & $33 / 16,5$ \\
\hline Amarela & $6 / 3,0$ \\
\hline \multicolumn{2}{|l|}{ Estado civil (n \%) } \\
\hline Solteiro & $43 / 21,5$ \\
\hline Casado & $113 / 56,5$ \\
\hline União estável & $12 / 6,0$ \\
\hline Divorciado & $24 / 12,0$ \\
\hline Viúvo & $8 / 4,0$ \\
\hline \multicolumn{2}{|l|}{ Situação no mercado de trabalho (n \%) } \\
\hline \multicolumn{2}{|l|}{ Inativos } \\
\hline Aposentado & $51 / 25,5$ \\
\hline Desempregado & $38 / 19,0$ \\
\hline Com auxílio doença & $35 / 17,5$ \\
\hline \multicolumn{2}{|l|}{ Ativos } \\
\hline Atuante no mercado formal & $59 / 29,5$ \\
\hline Atuante no mercado informal & $6 / 3,0$ \\
\hline Autônomo & $11 / 5,5$ \\
\hline
\end{tabular}

méricas podem afetar a mensuração pelo SG, efeito conhecido como variação irrelevante de construto $^{20-21}$. Mesmo assim, estas ferramentas têm sido consideradas como as mais adequadas para as análises de custo-utilidade, em função de serem teoricamente mais fundamentadas ${ }^{22}$.

Ao longo dos anos os sistemas multi-atributos, baseados em questionários (como o SF-6D), tiveram um papel importante na disseminação da aplicação das medidas de preferência, por tornarem mais práticos os processos de avaliação e
Tabela 2. Características clínicas dos pacientes com artrite reumatóide.

\begin{tabular}{|c|c|}
\hline & n \% ou Média (DP) \\
\hline \multicolumn{2}{|l|}{ Tempo de doença (anos) } \\
\hline $\mathrm{N}$ & 200 \\
\hline Média (DP) & $11,16(8,4)$ \\
\hline Mínimo - máximo & $1,00-42,00$ \\
\hline \multicolumn{2}{|l|}{ Classe funcional $^{1}$ (n \%) } \\
\hline I & 33,0 \\
\hline II & 38,5 \\
\hline III & 27,0 \\
\hline IV & 1,5 \\
\hline \multicolumn{2}{|c|}{ Número de articulações dolorosas } \\
\hline $\mathrm{N}$ & 200 \\
\hline Média (DP) & $5,56(6,9)$ \\
\hline Mínimo - máximo & $0-42,00$ \\
\hline \multicolumn{2}{|c|}{ Número de articulações edemaciadas } \\
\hline $\mathrm{N}$ & 200 \\
\hline Média (DP) & $7,35(6,7)$ \\
\hline Mínimo - máximo & $0-32,00$ \\
\hline \multicolumn{2}{|l|}{$\mathrm{EVA}^{3}$ de dor $(\mathrm{mm})$} \\
\hline $\mathrm{N}$ & 200 \\
\hline Média / DP & $41,42(25,1)$ \\
\hline Mínimo - máximo & $0-100,00$ \\
\hline \multicolumn{2}{|c|}{ Presença de deformidades ( $\mathrm{n} \%$ ) } \\
\hline Não & 27,0 \\
\hline Sim & 73,0 \\
\hline \multicolumn{2}{|c|}{$\begin{array}{l}\text { Presença de manifestações } \\
\text { extra-articulares (n \%) }\end{array}$} \\
\hline Não & 74,5 \\
\hline Sim & 25,5 \\
\hline \multicolumn{2}{|l|}{$\mathrm{HAQ}^{2}$} \\
\hline $\mathrm{N}$ & 199 \\
\hline Média (DP) & $1,02(0,7)$ \\
\hline Mínimo - máximo & $0-2,63$ \\
\hline \multicolumn{2}{|c|}{ Presença de cuidador ( $\mathrm{n} \%$ ) } \\
\hline Não & 75,5 \\
\hline Sim & 24,5 \\
\hline \multicolumn{2}{|l|}{$\mathrm{EVA}^{4}$ de $\mathrm{EGS}(\mathrm{cm})$} \\
\hline $\mathrm{N}$ & 200 \\
\hline Média (DP) & $67,30(20,7)$ \\
\hline Mínimo - máximo & $0-10,00$ \\
\hline
\end{tabular}

${ }^{1}$ Classe funcional pelo ACR: I = completamente capaz de realizar atividades de vida diária; II = limitado para atividades não vocacionais; III = limitado para atividades vocacionais e não vocacionais; IV = limitado para atividades de autocuidado, vocacionais e não vocacionais; ${ }^{2} \mathrm{HAQ}=$ Health Assessment Questionaire; ${ }^{3}$ EVA de dor $=$ Escala Visual Analógica de dor (obtida a partir do HAQ), onde $0=$ sem dor e $100=$ dor intensa; ${ }^{4}$ EVA de EGS = Escala Visual Analógica de estado geral de saúde (obtida a parti do EuroQol), onde $0=$ pior estado de saúde imaginável e $100=$ o melhor estado de saúde imaginável.

por sofrerem menos influência do estado cognitivo e das condições sócio-econômicas dos indivíduos avaliados. Entretanto, alguns autores têm 
Tabela 3. Média dos domínios e sumários do SF-36 e das medidas de preferências de pacientes com artrite reumatóide.

\begin{tabular}{|c|c|c|c|c|}
\hline & Média & $\mathrm{DP}$ & Mínimo & Máximo \\
\hline \multicolumn{5}{|l|}{ Domínio $^{1}$} \\
\hline Capacidade funcional & 47,97 & 25,6 & 0,00 & 100,00 \\
\hline Limitação por aspectos físicos & 43,62 & 43,5 & 0,00 & 100,00 \\
\hline Dor & 51,28 & 22,8 & 0,00 & 100,00 \\
\hline Estado geral de saúde & 52,03 & 17,2 & 5,00 & 92,00 \\
\hline Vitalidade & 55,00 & 19,9 & 5,00 & 100,00 \\
\hline Limitação por aspectos sociais & 71,46 & 25,9 & 13,00 & 100,00 \\
\hline Limitação por aspectos emocionais & 61,33 & 44,4 & 0,00 & 100,00 \\
\hline Saúde mental & 63,74 & 20,4 & 12,00 & 100,00 \\
\hline \multicolumn{5}{|l|}{ Sumário ${ }^{2}$} \\
\hline Físico & 36,20 & 11,1 & 14,27 & 61,82 \\
\hline Mental & 48,12 & 9,7 & 21,15 & 68,04 \\
\hline $\mathrm{EVA}^{3}$ & 0,63 & 0,2 & 0,03 & 0,98 \\
\hline $\mathrm{TTO}^{4}$ & 0,86 & 0,2 & 0,00 & 1,00 \\
\hline $\mathrm{SG}^{5}$ & 0,76 & 0,3 & 0,01 & 1,00 \\
\hline EQ-5D ${ }^{6}$ & 0,65 & 0,3 & $-0,48$ & 1,00 \\
\hline SF-6D ${ }^{7}$ & 0,81 & 0,1 & 0,41 & 0,99 \\
\hline
\end{tabular}

${ }^{1} 0=$ pior estado de saúde e $100=$ melhor estado de saúde; ${ }^{2}$ média da população americana $=50, \mathrm{DP}=10 ;{ }^{3} \mathrm{EVA}=$ Escala Visual Analógica, onde $0=$ pior estado de saúde imaginável e $1=$ melhor estado de saúde imaginável; ${ }^{4} \mathrm{TTO}=$ Time Trade-Off, onde $0=$ pior estado de saúde imaginável e $1=$ melhor estado de saúde imaginável; ${ }^{5} \mathrm{SG}=$ Standard Gamble, onde $0=$ pior estado de saúde imaginável e 1 = melhor estado de saúde imaginável; ${ }^{6} \mathrm{EQ}-5 \mathrm{D}=$ The Euroqol Index of Health-Related Quality of Life, onde $0,594=$ pior estado de saúde imaginável e $1=$ melhor estado de saúde imaginável; ${ }^{7} \mathrm{SF}-6 \mathrm{D}=$ The Short-Form 6 Dimensions, onde $+0,291$ = pior estado de saúde imaginável e 1 = melhor estado de saúde imaginável.

Tabela 4. Coeficientes de correlação de Pearson entre as medidas diretas de preferência por estados de saúde, o EQ-5D e o SF-6D, em pacientes com artrite reumatóide.

\begin{tabular}{lcrrrr}
\hline & SF-6D & EQ-5D & EVA & TTO & SG \\
\hline SF-6D $^{1}$ & 1,00 & $0,66^{* *}$ & $0,44^{* *}$ & $0,22^{* *}$ & $0,22^{* *}$ \\
EQ-5D $^{2}$ & & 1,00 & $0,49^{* *}$ & $0,19^{* *}$ & 0,11 \\
EVA $^{3}$ & & & 1,00 & $0,22_{* *}$ & 0,06 \\
TTO $^{4}$ & & & & 1,00 & $0,34^{* *}$ \\
SG $^{5}$ & & & & & 1,00 \\
\hline
\end{tabular}

${ }^{* *} \mathrm{p}<0,01 ;{ }^{1} \mathrm{SF}-6 \mathrm{D}=$ The Short-Form 6 Dimensions; ${ }^{2} \mathrm{EQ}-5 \mathrm{D}$

$=$ The Euroqol Index of Health-Related Quality of Life;

${ }^{3} \mathrm{EVA}=$ Escala Visual Analógica; ${ }^{4} \mathrm{TTO}=$ Time Trade-off;

${ }^{5} \mathrm{SG}=$ Standard Gamble.

questionado a baixa congruência entre os métodos indiretos (baseados em questionários) em relação aos métodos diretos, chegando a considerar inaceitável a variação nos resultados obtidos por diferentes métodos ${ }^{23-24}$.

Em relação à praticidade, os dados obtidos pelo presente estudo confirmam os já disponíveis na literatura, que revelam uma maior taxa de completude dos questionários administrados por entrevista em relação às técnicas de mensu- ração direta de preferências ${ }^{25-26}$. Em nosso estudo, quase todos os indivíduos conseguiram completar as avaliações baseadas em questionários (SF-6D, EQ-5D e HAQ), mas em relação às baseadas em técnicas de valoração (EVA, TTO e SG), uma parcela maior dos indivíduos não conseguiu completar a mensuração.

Podemos observar não só a variação entre as médias obtidas para as medidas diretas de preferência, com as medidas de EVA revelando valores mais baixos que as de TTO e SG; como também uma variação da média das preferências obtidas pelo SF-6D e pelo EQ-5D. Em nosso estudo os valores obtidos para SG foram menores que os de TTO, contradizendo em parte a literatura científica, que destaca a técnica de SG como geradora de valores mais altos de preferência, seguida de modo decrescente pelas técnicas de TTO e de EVA ${ }^{27}$. Este achado pode ser em parte explicado por maiores dificuldades de compreensão da técnica de SG em face ao baixo nível de escolaridade da amostra, mas resultados contraditórios já foram relatados previamente ${ }^{28,29}$.

Quando comparamos o estudo de Kaplan e colaboradores com os nossos dados, observamos correlações muito parecidas entre as preferências obtidas pelo SF-6D e as obtidas pelo EQ- 
$5 \mathrm{D}^{11}$. Nos estudos que avaliaram outras doenças, também observamos boas correlações entre diferentes medidas indiretas de preferência quando comparadas a medidas derivadas do SF$36^{11,24-26}$. De modo semelhante ao demonstrado em nosso estudo, em outras publicações, também as correlações destes métodos de derivação exibem resultados inferiores quando comparadas a medidas diretas (EVA, TTO, SG) ${ }^{10,25}$.

As altas correlações do SF-6D com o EQ-5D permitem notar um comportamento característico das correlações obtidas para o SF-6D, quando comparadas às diferentes medidas de preferência. Notamos que para todos os coeficientes, os menores valores foram obtidos sucessivamente para SG, seguidos de modo crescente para os obtidos em relação ao TTO, EVA e EQ-5D. Este comportamento chama a atenção para o já salientado por Prieto e Sacristán quanto ao fato de medidas de preferência obtidas através de questionários apresentarem características mais próximas de índices que sumarizam perfis de saúde, do que de medidas que refletem a preferência social por estados de saúde 4 . Também neste sentido, Revicki e Kaplan sugerem que a alta concordância entre medidas de preferência baseadas em questionários, como o SF-36, e as medidas específicas de qualidade de vida para determinadas doenças, deve-se em parte à sobreposição de questões presentes nestes instrumentos ${ }^{22}$.

Assim, as altas correlações entre o SF-6D e o EQ-5D são esperadas e constituem um importante aspecto na avaliação da validade do instrumento em estudo. Estas altas correlações também podem ser explicadas na medida em que ambos os instrumentos apresentam sistemas descritivos de estados de saúde, baseados em um conceito similar de qualidade de vida, que inclui o bem-estar físico, mental e social ${ }^{29}$. Além disso, o EQ-5D e o SF-6D foram desenvolvidos a partir de um modelo aditivo similar, em que os fatores relevantes que compõem a equação preditiva são somados para a obtenção do escore final ${ }^{28}$.

Em relação às dimensões do SF-6D acreditamos que, assim como acontece com o SF-36, a escala de limitações por aspectos físicos seja a mais sensível ao impacto das doenças crônicas, conforme descrito em diferentes estudos ${ }^{1,15}$. Entretanto, como o SF-6D, diferente do SF-36, apresenta um escore único, que sumariza as diferentes escalas, esta avaliação torna-se difícil de ser realizada em estudos empíricos.

De uma maneira geral, o SF-6D, enquanto medida genérica de qualidade de vida, possibilita aplicações diversas: rastreio e monitoramento de problemas psicossociais no cuidado individual, estudos populacionais sobre percepção de estados de saúde, auditoria médica, medidas de resultados em serviços de saúde, ensaios clínicos e análises econômicas ${ }^{30}$. Suas principais limitações estão relacionadas à sua utilização em contextos específicos a determinadas doenças, populações ou funções (capacidade funcional, função sexual, etc). Nestas situações, os instrumentos específicos de qualidade de vida seriam mais adequados, dado que o SF-6D poderia não ser clinicamente sensível a mudanças nas condições específicas de saúde dos grupos avaliados.

Por fim, algumas limitações deste estudo devem ser destacadas. Em primeiro lugar, a amostra selecionada a partir de um centro de referência pode não ser uma boa representação do universo de pacientes com artrite reumatóide. Em segundo lugar, a baixa escolaridade e o baixo nível sócio-econômico podem ter prejudicado a aplicação das técnicas mais complexas como o TTO e o SG. Em terceiro lugar, neste estudo não avaliamos a responsividadade dos algoritmos às mudanças no quadro clínico da doença ao longo do tempo. Entretanto, o estudo de Kaplan e colaboradores revelou boa responsividade dos algoritmos de Brazier e colaboradores ${ }^{11}$.

\section{Conclusões}

As medidas de preferência obtidas com o questionário SF-6D Brasil apresenta correlações significativas com as obtidas pelo EQ-5D e pelas técnicas de EVA, TTO e SG.

A versão mais atual do SF-6D, baseada no modelo de 2002, apresenta-se válida e representa uma opção de questionário para a avaliação de preferências em análises econômicas realizadas em nosso meio.

\section{Colaboradores}

AG Campolina, AB Bortoluzzo, MB Ferraze RM Ciconelli participaram igualmente de todas as etapas da elaboração do artigo. 


\section{Referências}

1. Ware JE, Sherbourne CD. The MOS 36-item short health survey (SF-36). I. Conceptual framework and item selection. Med Care 1992; 30(6):473-483.

2. Garrat AM, Schmidt L, Mackintosh A, Fitzpatrick R. Quality of life measurement: bibliographic study of patient assessed health outcome measures. BMJ 2002; 324(7351):1417-1421.

3. Torrance G W, Feeny D H. Utilities and QualityAdjusted Life Years. Intl J of Technology Assessment in Health Care 1989; 5(4):559-575.

4. Prieto L, Sacristán JA. Problems and solutions in calculating quality-adjusted life years (QALY's). Health Qual of Life Outcomes 2003; 1:80.

5. Nichol MB, Sengupta N, Globe DR. Evaluating quality-adjusted life years: estimation of the health utility index (HUI2) from the SF-36. Med Decis Making 2001; 21(2):105-112.

6. Torrance GW. Social preferences for health states: An empirical evaluation of three measurement techniques. Socioecon Plan Sci 1976; 10(3):128-136.

7. Kaplan R M, Bush J W, Berry C. Health status: Types of validity and the index of well-being. Health Serv Res 1976; 11(4):478-507.

8. Torrance GW. Designing and Conducting Cost-Utility Analyses. In: Spilker B, organizador. Quality of Life and Pharmacoeconomics in Clinical Trials. Philadelphia(USA): Lippincott-Raven Publishers; 1996. p. 1105-1111.

9. Brazier J, Usherwood T, Harper R, Thomas K. Deriving a preference-based single index from the UK SF-36 Health Survey. J Clin Epidemiol 1998; 51(11): 1115-28.

10. Lee TA, Hollingworth W, Sullivan SD. Comparison of directly elicited preference to preferences derived from the SF-36 in adults with asthma. Med Decis Making 2003; 23(4):323-334.

11. Kaplan RM, Groessl EJ, Sengupta N, Sieber WJ, Ganiats TG. Comparison of Measure Utility Scores and Imputed Scores From the SF-36 in Patients With Rheumatoid Arthritis. Med Care 2005; 43(1): 79-87.

12. Brazier JB, Roberts J, Deverill M. The estimation of a preference-based measure of health from the SF36. J Health Econ 2002; 21(2):271-292.

13. Gonçalves Campolina A, Bruscato Bortoluzzo A, Bosi Ferraz M, Mesquita Ciconelli R. Validity of the SF6D index in Brazilian patients with rheumatoid arthritis. Clin Exp Rheumatol 2009; 27(2):237-245.

14. Arnett FC, Edworthy SM, Bloch DA, McShane DJ, Fries JF, Cooper NS. The American Rheumatism Association 1987 revised criteria for the classification of rheumatoid arthritis. Arthritis Rheum. 1988; 31(3):315-324.

15. Ciconelli RM, Ferraz MB, Santos W, Meinão I, Quaresma MR. Tradução para a língua portuguesa e validação do questionário genérico de avaliação de qualidade de vida SF-36 (Brasil SF-36). Revista Brasileira de Reumatologia 1999; 39(3):143-150.

16. Wolfe F, Kleinheksel SM, Cathey MA, Hawley DJ, Spitz PW, Fries JF. The clinical value of the Stanford Health Assessment Questionnaire functional disability index in patients with rheumatoid arthritis. $J$ Rheumatol. 1988; 15(10):1480-1488.
17. Kind P. The performance characteristics of EQ-5D, a measure of health related quality of life for use in technology assessment [abstract]. Annual Meeting of International Society of Technology Assessment in Health Care 1997; 13(5):81.

18. Dolan P. Modeling Valuations for Euroqol Health States. Med Care 1997; 35(11):1095-1108.

19. Bennett KJ, Torrance GW. Measuring Health State Preferences and Utilities: Rating Scale, Time TradeOff, and Standard Gamble Techniques. In: Spilker B, editor. Quality of Life and Pharmacoeconomics in Clinical Trials. Philadelphia(USA): LippincottRaven Publishers; 1996. p. 253-265.

20. Lenert L, Kaplan RM. Validity and Interpretation of preference-based measures of health-related quality of life. Med Care 2000; 28(Suppl. 2):138-150.

21. Torrance GW, Furlong W, Feeny D. Health utility estimation. Expert Rev Pharmacoeconomics Outcomes Res. 2002; 2(2):99-108.

22. Revicki D, Kaplan R. Relationship between psychometric and utility-based approaches to the measurement of health-related quality of life. Qual Life Res 1993; 2(6):477-487.

23. Ferraz MB, Oliveira LM, Atra E, Bennett K. Performance of the McMaster Rheumatoid Arthritis Measurement Questionaire in Brazilian Patients. J Rheumatol 1992; 19(12):1994-1995.

24. Longworth L, Bryan S. An empirical comparison of EQ-5D and SF-6D in liver transplant patients. Health Econ 2003; 12(2):1061-1067.

25. Hollingworth W, Deyo RA, Sullivan SD, Emerson SS, Gray DT, Jarvik JG. The practicality and validity of directly elicited and SF-36 derived health state preferences in patients with low back pain. Health Econ 2002; 11(1):71-85.

26. Bosch JL, Hunink MG. The relationship between descriptive and valuational quality of life measures in patients with intermittent claudication. Med Decis Making 1996; 16(6):217-25.

27. O' Brien BJ, Spath M, Blackhouse G, Severens JL, Dorian P, Brazier J. A view from the bridge: agreement between the SF-6D utility algorithm and the Health Utilities Index. Health Econ 2003; 12(11): 975-981.

28. Kopec JA, Willison KD. A comparative review of four preference-weighted measures of health-related quality of life. J Clin Epidemiol 2003; 56(4):317-325.

29. Conner-Spady B, Suarez-Almazor ME. Variation in the estimation of quality-adjusted life-years by different preference-based instruments. Med Care 2003; 41(7):791-801.

30. Fitzpatrick R, Fletcher A, Gore S, Jones D, Spiegelhalter D, Cox D. Quality of life measures in health care. I: Applications and issues in assessment BMJ 1992; 305(6861):1074-1077.

Artigo apresentado em 03/03/2010

Aprovado em 16/03/2010

Versão final apresentada em 10/04/2010 